\title{
Honeycomb-inspired design of ultrafine SnO2@C nanospheres embedded in carbon film as anode materials for high performance lithium- and sodium-ion battery
}

Xiang Ao, J ianjun Jiang, Yunjun Ruan, Zhishan Li, Yi Zhang, Jianwu Sun and Chundong Wang

The self-archived version of this journal article is available at Linköping University Electronic Press:

http:/ / urn.kb.se/ resolve?urn=urn:nbn:se:liu:diva-139176

N.B.: When citing this work, cite the original publication.

Ao, X., Jiang, J ., Ruan, Y., Li, Z., Zhang, Yi, Sun, J., Wang, C., (2017), Honeycomb-inspired design of ultrafineSnO2@C nanospheres embedded in carbon film as anode materials for high performance lithium- and sodium-ion battery, J ournal of Power Sources, 359, 340-348.

https:// dx.doi.org/ 10.1016/j.jpowsour.2017.05.064

Original publication available at:

https:// dx.doi.org/ 10.1016/j.jpowsour.2017.05.064

Copyright: Elsevier

http://www.elsevier.com/

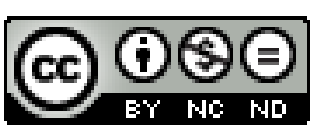




\section{Honeycomb-inspired design of ultrafine $\mathrm{SnO}_{2} @ \mathrm{C}$ nanospheres embedded}

in carbon film as anode materials for high performance lithium- and

\section{sodium-ion battery}

Xiang Ao ${ }^{\text {a }}$, Jianjun Jiang ${ }^{\mathrm{a}, * *}$, Yunjun Ruan ${ }^{\mathrm{a}}$, Zhishan Li ${ }^{\mathrm{a}}$, Yi Zhang ${ }^{\mathrm{b}}$, Jianwu Sun ${ }^{\mathrm{c}}$,

Chundong Wang ${ }^{\text {a, }} \mathrm{d}^{*}$

a School of Optical and Electronic Information, Huazhong University of Science and Technology, Wuhan 430074, P.R. China

${ }^{\mathrm{b}}$ School of Chemical Engineering and Pharmacy, Wuhan Institute of Technology, Wuhan, 430073, P.R. China

c Department of Physics, Chemistry and Biology (IFM), Linköping University, Linköping 58183, Sweden

${ }^{\mathrm{d}}$ Key Laboratory of Nanodevices and Applications, Suzhou Institute of Nano-Tech and Nano-Bionics, Chinese Academy of Sciences, Suzhou, 215123, P.R. China

* Corresponding author

** Corresponding author

E-mail: apcdwang@hust.edu.cn (C. Wang), jiangjj@mail.hust.edu.cn (J. Jiang),

Tel./fax: +86-27-8755 9279 


\section{ABSTRACT:}

Tin oxide $\left(\mathrm{SnO}_{2}\right)$ has been considered as one of the most promising anodes for advanced rechargeable batteries due to its advantages such as high energy density, earth abundance and environmental friendly. However, its large volume change during the Li-Sn/Na-Sn alloying and de-alloying processes will result in a fast capacity degradation over a long term cycling. To solve this issue, in this work we design and synthesize a novel honeycomb-like composite composing of carbon encapsulated $\mathrm{SnO}_{2}$ nanospheres embedded in carbon film by using dual templates of $\mathrm{SiO}_{2}$ and $\mathrm{NaCl}$. Using these composites as anodes both in lithium ion batteries and sodium-ion batteries, no discernable capacity degradation is observed over hundreds of long term cycles at both low current density $\left(100 \mathrm{~mA} \mathrm{~g}^{-1}\right)$ and high current density (500 mA $\left.\mathrm{g}^{-1}\right)$. Such a good cyclic stability and high delivered capacity have been attributed to the high conductivity of the supported carbon film and hollow encapsulated carbon shells, which not only provide enough space to accommodate the volume expansion but also prevent further aggregation of $\mathrm{SnO}_{2}$ nanoparticles upon cycling. By engineering electrodes of accommodating high volume expansion, we demonstrate a prototype to achieve high performance batteries, especially high-power batteries.

Keywords: Tin oxide; Honeycomb-inspired; Lithium ion batteries; sodium-ion batteries 


\section{Introduction}

With the continuous increase of the consumption of fossil fuels for human's modern life and industry production, the world now is suffering from a global energy challenge and serious environmental issues. This has stimulated a worldwide interest to explore clean and renewable energy sources in a large scale application as alternatives, such as harvest of solar, wind and tide energy. In this regard, one of the most important things that people are facing now is how to effectively store the intermittent renewable energies [1]. Lithium ion battery (LIB), as an electrochemical energy storage device, is one of the most powerful and promising rechargeable energy storage device due to its high energy density, long lifespan, and low self-discharge nature [2-5]. Nevertheless, due to the limited natural storage of lithium resources, immoderately pursue of mass production of Li-ion batteries have been suggested to be unrealistic in the long run. As an alternative, sodium ion battery has recently attracted ever-increasing attention owing to its high availability on the earth's crust (42.3 times vs. Li percentage) [6], and its similar chemical properties to lithium. However, compared with Li-ion battery, it is still a challenge to develop the Na-ion batteries since Na has larger atom size, larger atom weight and unfavorable redox potential of sodium upon charging/discharging [7]. Therefore, few materials can be used as host matrixes which could accommodate sodium ions and allow reversible $\mathrm{Na}$ insertion and extraction processes. Numerous materials which are qualified as matrixes for Li-ion batteries might not be suitable for the Na-ion batteries. For example, graphite, which is the most widely used as anode material in commercial LIBs, has been disclosed to hardly accommodate sodium ions by early investigations [8, 9].

On the other hand, due to the abundance and environmentally benign nature, Tin and Tin 
based materials have demonstrated outstanding performance as anode in LIBs [10, 11]. Among them, $\mathrm{SnO}_{2}$, which possesses a theoretic capacity of $782 \mathrm{mAhg}^{-1}$, has been considered as one of the most promising candidates to substitute the commercialized graphite anode [1, 12]. To date, only a few studies have reported that $\mathrm{SnO}_{2}$ can reversibly alloy with $\mathrm{Na}$ $\left(4 \mathrm{SnO}_{2}+31 \mathrm{Na}^{+}+31 \mathrm{e}^{-}=\mathrm{Na}_{15} \mathrm{Sn}_{4}+8 \mathrm{Na}_{2} \mathrm{O}\right)[13,14]$. Theoretically, such alloy can deliver a capacity of 1378 mAhg- 1 and thus can be potentially used as a superb anode material for Na-ion battery with the merit of low price and environment benign. Upon the insertion of $\mathrm{Li}^{+}$ and/or $\mathrm{Na}^{+}$into $\mathrm{SnO}_{2}$, huge volume expansion of the host materials is inevitably accompanied, leading to the rapid capacity degradation due to the pulverization and aggregation of the electrode active materials [15]. To solve this volume change problem and effectively stabilize $\mathrm{SnO}_{2}$ nanoparticles, different methods such as configuration of $\mathrm{C} / \mathrm{SnO}_{2}$ composites [16], $\mathrm{SnO}_{2}$ /nanotubes [14] and $\mathrm{SnO}_{2}$-RGO $[17,18]$ have been proposed. However, it is still challenging to achieve high-performance LIBs and sodium-ion batteries (SIBs).

In this work, we propose a novel and scalable approach for the preparation of honeycomb-like composites, i.e. $\mathrm{SnO}_{2} @ \mathrm{C}$ nanospheres embedded in carbon film by a two-step process using silica nanospheres and sodium chloride $(\mathrm{NaCl})$ as templates and demonstrate that such a novel structure can provide enough buffer spaces for volume expansion during $\mathrm{Li}^{+} / \mathrm{Na}^{+}$uptake and release processes thus promoting the performance of LIBs and SIBs. The electrochemical performance in LIB and SIB has been evaluated by assembling the active material into a half-cell. We found that cavities are formed on the nanosphere surface and the nanospheres are densely embedded in carbon sheets, forming a honeycomb-like architecture. The carbon film as a support for carbon encapsulated $\mathrm{SnO}_{2}$ 
nanospheres would effectively enhance the electrical conductivity, while the cavity on the sphere surface and the hollow nature of our designed structure would provide enough buffer space to accommodate the volume expansion of anode material over cycling. Moreover, besides of its function as physical buffering layer as well as restrictor for the growth of solid electrolyte interphase (SEI) layer, the encapsulated carbon shells can also work as conducing layer to promote the transport of electrons and ions. Meanwhile, the $\mathrm{SnO}_{2}$ inside the carbon spheres was ultrafine, with dimensions of $<10 \mathrm{~nm}$, can endure the $\mathrm{Li}^{+} / \mathrm{Na}^{+}$insertion and extraction. Finally we demonstrate the excellent rate capability and long cycle life using our as-prepared nanostructure as an anode for Li-ion and Na-ion batteries. .

\section{Experimental section}

\subsection{Synthesis of $\mathrm{SiO}_{2}$ nanospheres.}

$\mathrm{SiO}_{2}$ nanoparticles was prepared with Stöber method [19]. In a typical procedure, $4.5 \mathrm{~mL}$ Tetraethyl orthosilicate was added into a mixed solution containing $61.75 \mathrm{~mL}$ ethanol and $24.75 \mathrm{~mL}$ deionized (DI) water under vigorous stirring. Then $9 \mathrm{~mL}$ ammonia solution was added into the above mixture drop by drop. The mixture was stirred at room temperature for 8 h. Subsequently, the product was centrifuged and washed for several times with ethanol and DI water. Finally, the white $\mathrm{SiO}_{2}$ nanospheres were obtained after drying at $60{ }^{\circ}$ Cover night.

2.2. Synthesis of $\mathrm{SiO}_{2} @ \mathrm{SnO}_{2}$ core-shell nanospheres.

$\mathrm{SnO}_{2}$ was deposited onto the $\mathrm{SiO}_{2}$ template surface by a hydrothermal method. In detail, $0.04 \mathrm{~g}$ of $\mathrm{SiO}_{2}$ nanospheres were homogeneously dispersed in a mixed solution of ethanol and DI water (37.5 vol\% of ethanol, $60 \mathrm{~mL}$ ) to form a white suspension by continuous ultrasonication and its further stirring. $0.3 \mathrm{~g}$ of $\mathrm{K}_{2} \mathrm{SnO}_{3} \cdot 3 \mathrm{H}_{2} \mathrm{O}$ and $1.8 \mathrm{~g}$ of urea were added to 
the above suspension under constant vigorous stirring. After stirring for 5 minutes, the suspension was transferred to a $100 \mathrm{~mL}$ Teflon lined stainless steel autoclave, which was then heated to $170^{\circ} \mathrm{C}$ and kept at this temperature for $18 \mathrm{~h}$. The product was centrifugally washed with DI water and ethanol for over five times before drying at $60{ }^{\circ} \mathrm{C}$ overnight. Finally, the white $\mathrm{SiO}_{2} @ \mathrm{SnO}_{2}$ nanospheres were obtained.

\subsection{Preparation of honeycomb-like composites}

$0.2 \mathrm{~g}$ of the as-prepared $\mathrm{SiO}_{2} @ \mathrm{SnO}_{2}$ nanospheres were dispersed in $67 \mathrm{~mL}$ DI water by ultrasonication, and then $0.1 \mathrm{~g}$ glucose and $10 \mathrm{~g} \mathrm{NaCl}$ were added to the above homogeneously solution under stirring. After the glucose and $\mathrm{NaCl}$ were completely dissolved, the suspension was heated to $100^{\circ} \mathrm{C}$ under robust stirring to evaporate the water until it become powder. The as-obtained product was placed in a corundum and heated to $650{ }^{\circ} \mathrm{C}$ and hold for $3 \mathrm{~h}$ in a furnace in Ar atmosphere. After cooling down naturally, the sample was subjected to a $2 \mathrm{M} \mathrm{NaOH}$ solution at $50^{\circ} \mathrm{C}$ for $8 \mathrm{~h}$ to remove the $\mathrm{SiO}_{2}$ template, and then filtrated and washed with DI water to remove $\mathrm{NaOH}$ and $\mathrm{NaCl}$ until $\mathrm{pH}=7$. Finally, the honeycomb-like composites, i.e. $\mathrm{SnO}_{2} @ \mathrm{C}$ nanospheres embedded in carbon films were obtained after being dried at $60{ }^{\circ} \mathrm{C}$ for one night.

For comparison, more glucose was added in a parallel experiment using a similar procedure as described above to prepared samples with different carbon content, i.e. the honeycomb-like composites-less.

\subsection{Characterizations}

The morphology and microstructure of samples were characterized using a filed-emission scanning electron microscope (FE-SEM, Nova NanoSEM 450) and a transmission electron 
microscopy (TEM, Tecnai G2 20, operated with accelerating voltage of $200 \mathrm{KV}$ ), and electron energy loss spectroscopy (EELS, attached to TEM). The crystalline structures of the composites were characterized by X-ray diffraction (XRD, X'Pert PRO). Thermogravimetrc analysis (Pyris1 TGA) was carried out under air from room temperature to $800{ }^{\circ} \mathrm{C}$ with a heating rate of $10^{\circ} \mathrm{C} / \mathrm{min}$. XPS spectra were measured by an AXIS-ULTRA DLD-600W spectrometer. Raman spectra were collected on Raman microscopes (LabRAM HR800) under the excitation of the laser line of $532 \mathrm{~nm}$. Brunauer-Emmett-Teller (BET) surface area was determined by a Micromeritics Tristar II 3020 instrument.

\subsection{Electrochemistry Measurements.}

CR2016 coin-type cells assembled in an argon-filled glove box (MBraun Lab Master PRS257) were utilized to test the electrochemistry performance of the samples. The working electrodes were prepared by mixing of the active materials, acetylene black and polyvinylidene fluoride (PVDF) binder in N-methylpyrrolidone with the mass ratio of 8:1:1. The resultant slurry was then uniformly pasted on a copper foil with doctor blade and dried at $100{ }^{\circ} \mathrm{C}$ overnight under vacuum. The electrolyte for LIBs in this work was a solution of $1.0 \mathrm{M}$ LiPF6 dissolved in a mixed solvent of ethylene carbonate, diethyl carbonate and dimethyl carbonate (1:1:1 in volume), and Celgard 2325 porous film was used as the separator. For SIBs, 1.0 $\mathrm{M} \mathrm{NaClO}_{4}$ dissolved in a mixed solvent of ethylene carbonate and diethyl carbonate (1:1 in volume) was selected as the electrolyte, and a glass fiber (Whatman) was used as the separator.

Galvanostatic charge/ discharge was tested at various rates via a battery tester (CT2001A, LAND), and cyclic voltammetry at the scan rate of $0.1 \mathrm{mV} \mathrm{s}^{-1}$ were performed on an 
electrochemical workstation (CHI 760E, CH Instruments Ins). All the specific capacities and the current densities used in this work were based on the total weight of the active materials.

\section{Result and discussion}
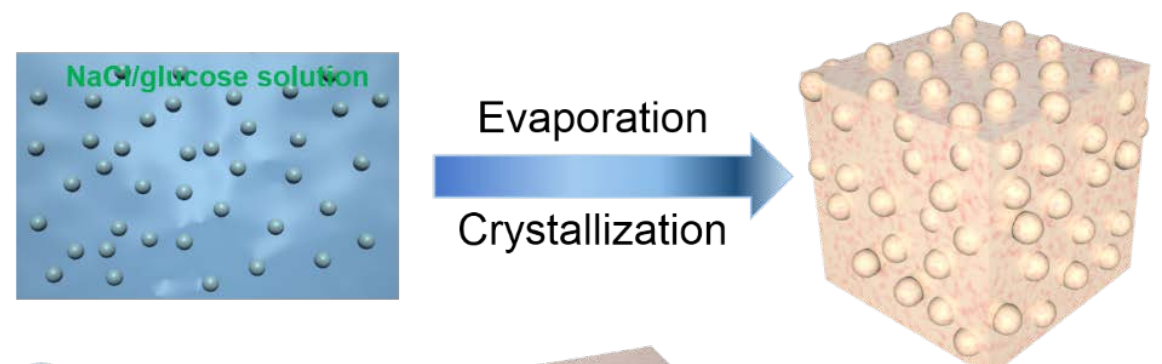

$\mathrm{SiO}_{2} @ \mathrm{SnO}_{2}$ nanosphere
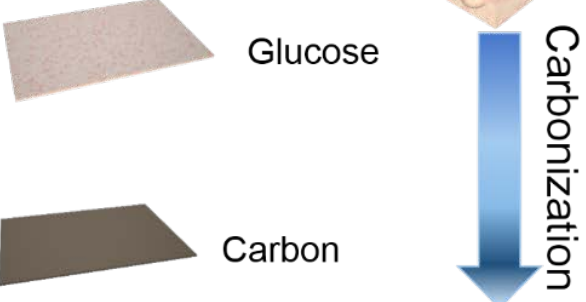

Hollow $\mathrm{SnO}_{2} @ \mathrm{C}$

Carbon

Dissolve
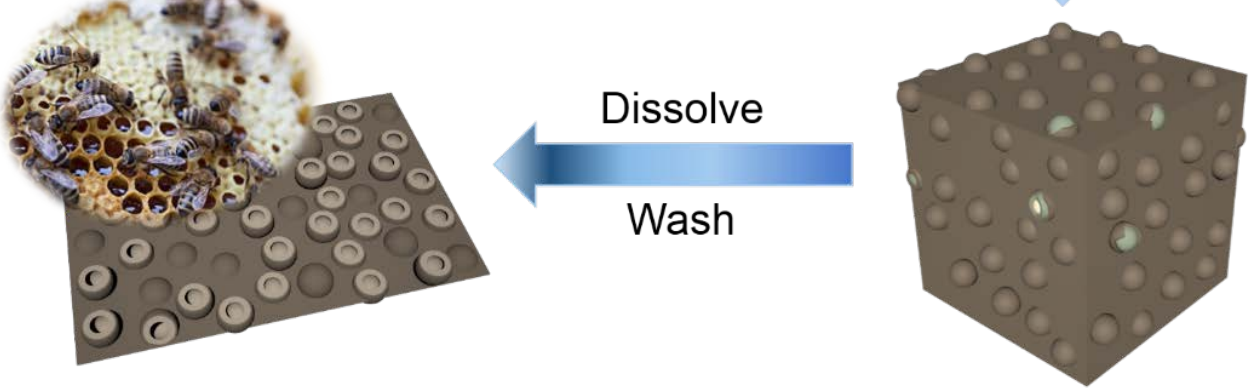

Fig. 1. Schematic illustration of the fabrication procedure of honeycomb-inspired $\mathrm{SnO}_{2} @ \mathrm{C}$ nanospheres embedded in carbon film structure.

The fabrication of $\mathrm{SnO}_{2} @ \mathrm{C}$ nanospheres embedded in carbon film is schematically depicted in Fig. 1. Firstly, $\mathrm{SnO}_{2}$ were grown on $\mathrm{SiO}_{2}$ nanospheres to form a core/shell structure (donated as $\mathrm{SiO}_{2} @ \mathrm{SnO}_{2}$ ) using a Stöber method and a simple hydrothermal process. The detail preparation process is described in the experimental section and the morphology of $\mathrm{SiO}_{2} @ \mathrm{SnO}_{2}$ is shown in Fig. S1a and b. The core/shell structure can be well identified in Fig. S1a, where a typical partial broken $\mathrm{SiO}_{2} @ \mathrm{SnO}_{2}$ is found. In a further enlarged SEM image, a 
rough surface of $\mathrm{SnO}_{2}$ is disclosed, suggesting $\mathrm{SnO}_{2}$ shell should be composed of nanoparticles. Secondly, the $\mathrm{SiO}_{2} @ \mathrm{SnO}_{2}$ nanospheres were dispersed in $\mathrm{NaCl} /$ glucose solution, following which it was vigorous stirred continuously. Upon water evaporation, cubic $\mathrm{NaCl}$ crystals were formed with densely $\mathrm{SiO}_{2} @ \mathrm{SnO}_{2}$ nanospheres and glucose decorated on the surface (see Fig. S2) because of the face-centered cubic crystal structure nature of $\mathrm{NaCl}$ [20]. With a further carbonization treatment, glucose was converted to carbon and uniformly covered both on the surfaces of the $\mathrm{NaCl}$ template and the $\mathrm{SiO}_{2} @ \mathrm{SnO}_{2}$ nanospheres. Finally, the $\mathrm{SiO}_{2}$ cores were removed by dissolving in $\mathrm{NaOH}$ solution, and $\mathrm{NaCl}$ template was washed away by deionized (DI) water, obtaining the final product of SnO2@C nanospheres embedded in carbon film (designated as honeycomb-like composites).

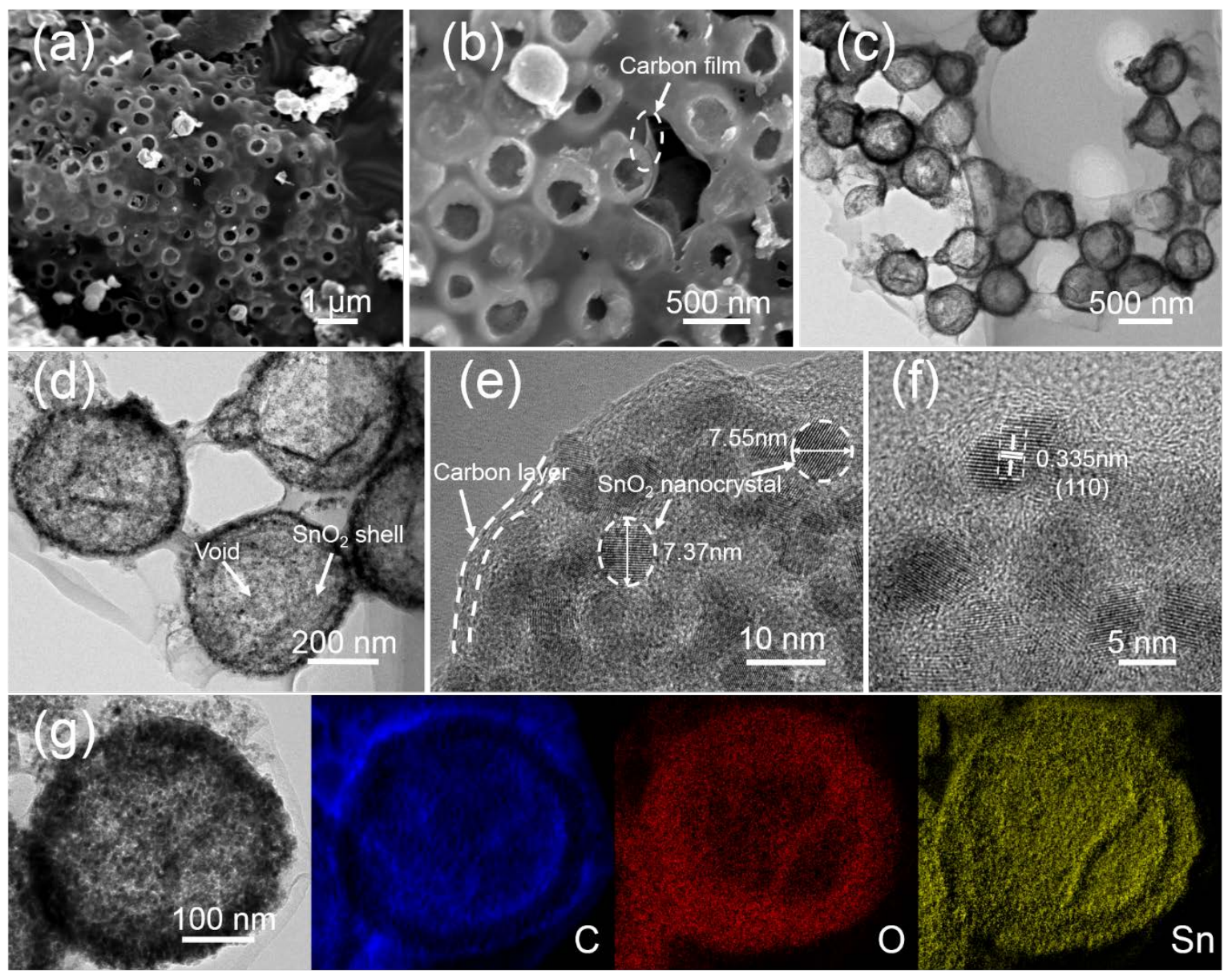

Fig. 2. (a, b) SEM, (c, d) TEM, (e, f) HRTEM and (g) EELS elemental mapping analysis 
images of honeycomb-like composites.

In Fig. 2a, the SEM image of the as-prepared products shows a typical honeycomb-like structure. A further magnified SEM image (Fig. 2b) reveals that the prepared honeycomb-like composites are composed of numerous broken nanospheres supported by a carbon film, which is quite similar to the honeycomb as depicted in Fig. 1. Fig. 2b shows one edge of a broken hole in the carbon sheet as marked by an arrow, indicating the supported carbon film is very thin. The diameter of nanosphere is $300-500 \mathrm{~nm}$. The formation of a honeycomb-like architecture instead of a bubble sheet configuration might be due to the fact that some $\mathrm{SnO}_{2}$ was partially dissolved during the dissolution process of $\mathrm{SiO}_{2}$. Due to the large surface tension effect as well as a mild chemical reaction, this dissolution behavior only happened on the top surface of the sphere, forming honeycomb-like structure. This assumption can be evidenced by the morphology change of the product before and after the $\mathrm{NaOH}$ treatment, as shown in Fig. S1a and c. It is seen that most $\mathrm{SiO}_{2} @ \mathrm{SnO}_{2}$ spheres before $\mathrm{NaOH}$ treatment are intact, while for the case of $\mathrm{SnO}_{2}$ after dissolving of $\mathrm{SiO}_{2}$, the void on the sphere is clearly observed (see Fig. S1d).

TEM measurement was carried out to further characterize the microstructure of our prepared honeycomb-like composites. In Fig. 2c and d, it shows that carbon encapsulated interconnected nanospheres that packed with loosely nanoparticles can be well recognized and the void in the nanospheres can be clearly observed. Due to the ultrasonic treatment during TEM sample preparation process, the supported carbon film was broken, while the nanospheres can still remain the sphere configuration, manifesting the robust nature of our 
designed honeycomb-like structure and the supporting carbon film is very thin. At one edge of a chosen $\mathrm{SnO}_{2} @ \mathrm{C}$ sphere, the thickness of the carbon shell is confirmed to be 2-4 nm (marked with dashed line and arrow in Fig. 2e, also seen in Fig. S3). Fig. 2e discloses that $\mathrm{SnO}_{2}$ nanocrystallines are densely and homogeneous decorated on the carbon shell, and the size of the $\mathrm{SnO}_{2}$ nanocrystallines are less than $10 \mathrm{~nm}$. High-resolution TEM (HRTEM) iage shown in the highlighted white dash box in Fig. $2 \mathrm{f}$ demonstrates the well-resolved lattice space of $0.335 \mathrm{~nm}$ (calculated from intensity profile of lattice in Fig. S5), indicating the (110) lattice planes of $\mathrm{SnO}_{2}$. To determine the element distribution in the honeycomb-like composites, electron energy loss spectroscopy (EELS) mappings were carried out. It shows that tin and oxygen elements were evenly distributed in the whole nanosphere, while an obvious blank ring was seen in carbon mapping image, suggesting that $\mathrm{SnO}_{2}$ was mainly decorated in the inner shell of carbon. This confirms the hollow core-shell structure by dissolving of $\mathrm{SiO}_{2}$ from SiO2@SnO2@C spheres.

Nitrogen isotherm adsorption - desorption measurement was implemented to access the porous characteristics of the as-prepared honeycomb-like composites. A typical type IV isothermal curve was seen in Fig. S5a, which is the characteristic of mesoporous materials [21]. The result shows that the specific surface area of the honeycomb-like nanostructure is $\sim 231.7 \mathrm{~m}^{2} \mathrm{~g}^{-1}$. Besides, a narrow pore size distribution centered at $31 \mathrm{~nm}$ was observed in the inset of Fig. S5a, evidencing that the carbon inner shell was loosely decorated with $\mathrm{SnO}_{2}$ nanocrystallines, instead of compact $\mathrm{SnO}_{2}$ thin film, which is also consistent with the TEM observation discussed aforementioned. 

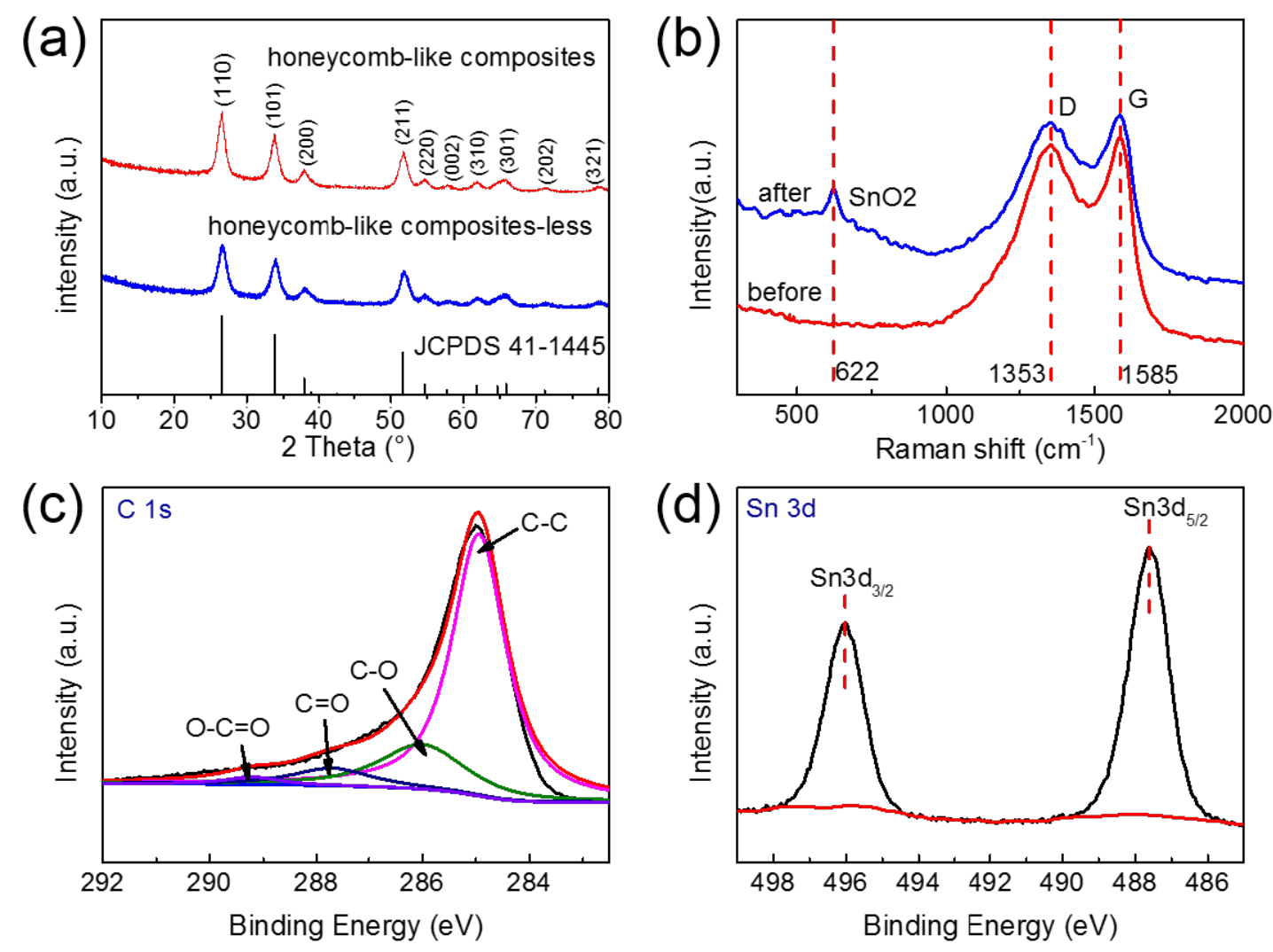

Fig. 3. (a) XRD patterns of the honeycomb-like composites, honeycomb-like composites-less and the standard XRD pattern of $\mathrm{SnO}_{2}$; (b) Raman spectra of honeycomb-like composites before and after removal of carbon by laser; (c, d) Core-level XPS spectra of C 1s and Sn 3d of the honeycomb-like composites.

XRD and Raman spectroscopy were recorded to access the structural characteristics of the honeycomb-like composites. Fig. 3a shows that the XRD patterns of the honeycomb-like composites can be well-indexed to tetragonal rutile-type $\mathrm{SnO}_{2}$ (JCPDS Card no. 41 - 1445; space group: P42/mnm (136); $a=b=4.738 \AA, c=3.187 \AA ; \alpha=\beta=\gamma=90^{\circ}$ ). The main diffraction peaks centered at $26.6^{\circ}, 33.9^{\circ}, 38.0^{\circ} 51.8^{\circ}$ and $54.8^{\circ}$ can be assigned to the (110), (101), (200), (211) and (220) planes of the rutile $\mathrm{SnO}_{2}$, respectively. Diffraction peaks of carbon were not observed in the XRD patterns due to the strong diffraction intensity of $\mathrm{SnO}_{2}$ and the very thin 
carbon films and shells. To evaluate the percentage of the carbon in the honeycomb-like composites, themogravimetric analysis (TGA) was implemented in air with a flow rate of 10 $\mathrm{ml} \mathrm{min}{ }^{-1}$. In detail, the sample was heated from 30 to $800{ }^{\circ} \mathrm{C}$ at a rate of $10{ }^{\circ} \mathrm{C} \mathrm{min}^{-1}$. An obvious weight loss starting at about $400^{\circ} \mathrm{C}$ is seen (Fig. S5b), indicating the combustion of carbon component upon the heating. This result shows that the content of carbon in the honeycomb-like composites should be 28.6 wt\%. For comparison, the honeycomb-like composites with more large percentage carbon content (termed as honeycomb-like composites-less) was also prepared. XRD results show the same pattern but a little bit weak intensity compared with that of the honeycomb-like composites (shown in Fig. 3a). And the content of the carbon in this case was found to be $43.6 \mathrm{wt} \%$ based on the weight loss upon combustion in air (see Fig. S5b).

Fig. 3b shows the Raman spectrum of the honeycomb-like composites, in which two strong peaks at 1583 and $1353 \mathrm{~cm}^{-1}$ are revealed. The $\mathrm{G}$ band at $1583 \mathrm{~cm}^{-1}$ is associated to the characteristic doubly degenerate $\mathrm{E}_{2 \mathrm{~g}}$ mode (iTO and LO) of graphite, i.e. the $\mathrm{sp}^{2}$ in plane phonon vibrations $[10,22,23]$. The $\mathrm{D}$ band at $1353 \mathrm{~cm}^{-1}$ is the characteristic of defects in graphite, assigning to strongly dispersed LO phonons around the $\mathbf{K}$ point activated in double resonance processes [24-26]. More specifically, a Kohn anomaly at $\mathbf{K}$ is suggested to be the true origin of the $\mathrm{D}$ band due to the highest optical branch starting from the $\mathbf{K}-\mathrm{A}_{1}$ ' mode, which has the biggest electron-phonon coupling among the $\mathbf{K}$ phonons [27]. Interestingly, it is seen that the intensity of the $G$ band is a little bit higher than that of $D$ band, i.e. $I_{D} / I_{G} \approx$ 0.97, indicating high graphitization of the counterpart carbon in our prepared honeycomb-like composites. Unfortunately, no Raman signals of $\mathrm{SnO}_{2}$ were detected. To solve this problem, 
$532 \mathrm{~nm}$ laser was employed to remove the carbon shell by illuminating the sample for a while. An explicit peak at $622 \mathrm{~nm}$ was appeared (Fig. 3b), which associates to the $\mathrm{A}_{1 \mathrm{~g}}$ mode of Raman active of $\mathrm{SnO}_{2}$ that vibrates in the plane perpendicular to the c-axis [28]. Again, this observation indicates that the $\mathrm{SnO}_{2} @ \mathrm{C}$ unit in the honeycomb-like composites is core/shell structure, being consist with the obtained TEM images.

The chemical composition of the honeycomb-like composites was further studied by XPS. A survey spectrum is depicted in Fig. S5c. As expected, elements of C, O, and Sn are co-existing in our sample. Fig. 3c shows the C core-level spectrum. After careful deconvolution and fitting, four peaks that centered at 284.9, 286.0, 287.7and $289.2 \mathrm{eV}$ are found, which are assigned to graphite-like $\mathrm{sp}^{2}-\mathrm{C}(\mathrm{C}-\mathrm{C})$, epoxy groups (C-O), carbonyl groups $(\mathrm{C}=\mathrm{O})$, and carboxyl groups $(\mathrm{COOH})$, respectively [5, 29, 30]. In Fig. 3d, the fine spectrum of Sn $3 d$ shows two peaks at 487.6 and $496.0 \mathrm{eV}$, corresponding to $\mathrm{Sn} 3 \mathrm{~d}_{5 / 2}$ and $\mathrm{Sn} 3 \mathrm{~d}_{3 / 2}$. Of note, Sn $3 d_{5 / 2}$ centered at $487.6 \mathrm{eV}$ is associated to $\mathrm{Sn}^{4+}$ form of $\mathrm{SnO}_{2}$,[29] while the distance of the two peaks of $8.4 \mathrm{eV}$ is in consistent with splitting energy of $\mathrm{SnO}_{2}$ that reported in literature [31]. Furthermore, O 1s XPS spectrum shown in Fig. S5d is also observed. Three peaks at 533.6, 532.5 and $531.5 \mathrm{eV}$ are identified by deconvolution and fitting, which might be related to the boding of $\mathrm{C}-\mathrm{O}, \mathrm{C}=\mathrm{O}$ and Sn-O, respectively. This further manifests the $\mathrm{Sn}$ atoms are in the form of $\mathrm{SnO}_{2}[32]$. 

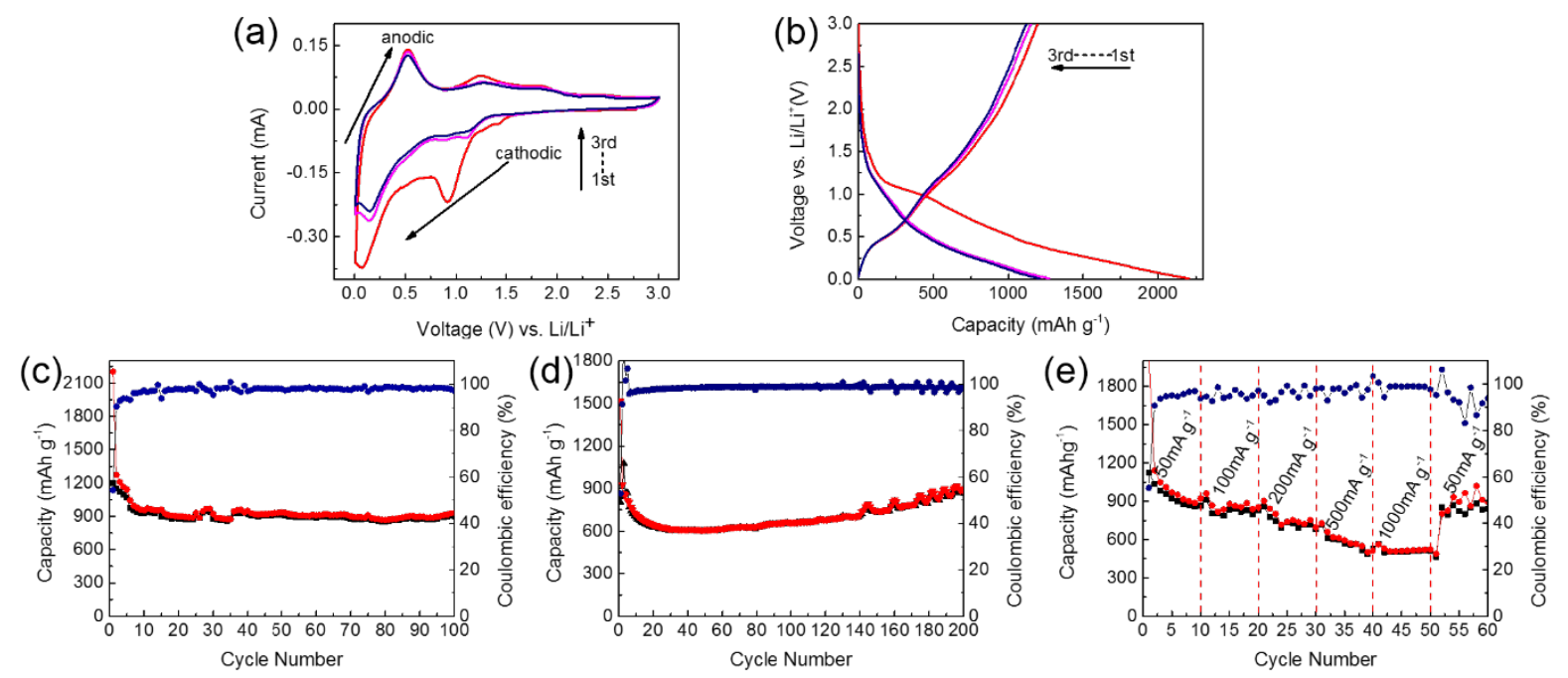

Fig. 4. (a) Cyclic voltammograms of the honeycomb-like composites anodes in LIBs; (b) Voltage profiles of the initial three cycles of the honeycomb-like composites anodes in LIBs; (c, d) Cycling performance and Coulombic efficiency of the honeycomb-like composites anodes in LIBs at current densities of $100 \mathrm{~mA} \mathrm{~g}^{-1}$ and $500 \mathrm{~mA} \mathrm{~g}^{-1}$; (e) Rate capabilities of the honeycomblike composites anodes in LIBs.

To evaluate the lithium storage capability, the honeycomb-like composites was assembled into a coin-type half-cell using Li metal foil as a counter electrode. Fig. 4a shows cyclic voltammograms (CV) of the honeycomb-like composites tested between 3.0 to $0 \mathrm{~V}$ at a scan rate of $0.1 \mathrm{mVs}^{-1}$. In the first scan, an obvious peak at $\sim 0.9 \mathrm{~V}$ is depicted, assigning to the reduction of $\mathrm{SnO}_{2}$ to $\mathrm{Sn}$ and the formation of $\mathrm{Li}_{2} \mathrm{O}$ (The detail chemical reaction can refer to eqn. 1 [33]). Upon further charging, the steep reduction peak below $0.5 \mathrm{~V}$ associates to $\mathrm{Li}$ Sn alloying processes (eqn. 2). A broad peak at $0.53 \mathrm{~V}$ in the anodic scan corresponds to the de-alloying of $\mathrm{Li}_{\mathrm{x}} \mathrm{Sn}$ [34], and the two additional broad oxidation peaks at $1.24 \mathrm{~V}$ and $1.84 \mathrm{~V}$ are possibly related to the partial reversible oxidation of $\mathrm{Sn}$ towards $\mathrm{SnO}_{2}$ [35]. By comparing 
the second/third cycle with the first one, it can be found that the peak at $0.9 \mathrm{~V}$ vanishes and tends to be stable after the first cycle, indicating the irreversible formation of SEI layer due to the decomposition of electrolyte. For comparison, Fig. S6a shows the CV test of honeycomb-like composites-less, which gives identical results of the oxidation/reduction peak positions and the peak variations upon charging/discharging.

$$
\begin{gathered}
\mathrm{SnO}_{2}+4 \mathrm{Li}^{+}+4 e^{-} \leftrightarrow \mathrm{Sn}+2 \mathrm{Li}_{2} \mathrm{O} \\
x \mathrm{Li}^{+}+\mathrm{Sn}+x e^{-} \leftrightarrow \mathrm{Li}_{x} \mathrm{Sn}
\end{gathered}
$$

Fig. 4b shows the galvanostatic charge-discharge voltage profiles of the honeycomb-like composites electrode for the first three cycles, at a current density of $100 \mathrm{mAg}^{-1}$ over a voltage range of $0.01-3.0 \mathrm{~V}$. In the first discharge profile, the voltage dropped quickly to $1.1 \mathrm{~V}$ and showed a discharge plateaus at $0.9-1.1 \mathrm{~V}$, assigning to the reduction of $\mathrm{SnO}_{2}$ to $\mathrm{Sn}$. The discharge plateaus ranging from $0.3-0.4 \mathrm{~V}$ is related to the $\mathrm{Li}-\mathrm{Sn}$ alloying towards formation of $\mathrm{Li}_{\mathrm{x}} \mathrm{Sn}$ alloy, which is consistent with the CV curves that discussed aforementioned. The honeycomb-like composite anode delivers a high discharge capacity of $2203 \mathrm{mAh} \mathrm{g}^{-1}$ and specific capacity of $1197 \mathrm{mAh} \mathrm{g}^{-1}$, yielding a Coulombic efficiency of $\sim 45.7 \%$. The large irreversible capacity in the first cycle could be caused by the formation of SEI on the electrode surface because high surface areas of our prepared honeycomb-like composites provide large contact area between the electrode and the electrolyte and consume large amount of $\mathrm{Li}^{+}$ions [5, 36]. Generally, the initial CE of hard carbon anodes are typically below $55 \%$ because of the formation of thick SEI layers [37], which is deemed as another possible reason. Besides, the functional groups on carbon surface might also contribute to the irreversible capability [5]. However, more possible behind reason may not be listed here and 
fully understood yet. In the second and third cycle, the discharge/charge capacities are 1274/1151 mAh g ${ }^{-1}, 1209 / 1122 \mathrm{mAh} \mathrm{g}^{-1}$, giving the corresponding coulombic efficiencies of 90.4\% and 92.8\%, respectively. The voltage profiles of the honeycomb-like composites-less shows similar results as seen in Fig. S6b.

Fig. 4c shows the cycling performance of the honeycomb-like composites measured at a current density of $100 \mathrm{~mA} \mathrm{~g}^{-1}$. Though decreasing in the initial several cycles, the delivered capacity tends to be stable in the following cycles. After 100 cycles, a specific capacity of $928.9 \mathrm{mAh} \mathrm{g}^{-1}$ is still maintained, which is over 2 times higher than the theoretical capacity of graphite (372 $\left.\mathrm{mAhg}^{-1}\right)$. Compared to the $12^{\text {th }}$ cycle (one randomly selected stable cycle), a capacity retention of $96.3 \%$ can be calculated for the $100^{\text {th }}$ cycle, indicating that nearly no capacity decaying was observed upon cycling. The excellent cyclic stability could be due to our unique designed honeycomb-like structure that allow $\mathrm{SnO}_{2}$ to be fully utilized in storing lithium ions. And the encapsulated carbon shells and supported carbon films provide amounts of efficient pathways for fast as well as efficiency electron/ion transfer [1]. Besides, the hollow unit of $\mathrm{SnO}_{2} @ \mathrm{C}$ together with its carbon membrane in the honeycomb-like configuration provides enough space to accommodate the volume expansion during the charging/discharging processes and prevents the further aggregation of $\mathrm{SnO}_{2}$ nanoparticles upon cycling [38-41]. Another possible reason for the high capacities and good stability could be the as-synthesized ultrafine $\mathrm{SnO}_{2}$ nanoparticles $(<10 \mathrm{~nm})$ embedded in the carbon shell, as it was reported in the literatures that engineering the size of metal oxide to nanoscales could effectively improve the electrochemical performance [42]. Additionally, it should be noted that the delivered stable capacity of $928.9 \mathrm{~mA} \mathrm{~g}^{-1}$ after 100 cycles is even higher than the 
theoretical capacity of $\mathrm{SnO}_{2}\left(781 \mathrm{mAhg}^{-1}\right)$. This result could be possibly due to the formation of SEI layer by decomposition of the electrolyte [43-45]. Such kind electrochemical behavior, namely charging/discharging capacity higher than the theoretical value, has been widely observed in other materials, particularly in transition metal oxide electrodes, and the reason has been assigned to the formation of gel-like organic layer on the surface of the porous structures over cycling [46, 47].

Encouraged by the excellent cycling stability, the honeycomb-like composites anodes were further assessed at a high current density of $500 \mathrm{~mA} \mathrm{~g}^{-1}$ and shown in Fig. 4d. Again, no capacity decay was observed, delivering high capacities of 662 and $881 \mathrm{mAh} \mathrm{g}^{-1}$ after 100 cycles and 200 cycles, respectively. Unlike the usually decay behavior [48] and/or the stable cycling performance at low current density that given in Fig. 4c, the delivered capacity keeps increasing over cycling after 70 cycles. We speculate the reason could be assigned to the activation process of the $\mathrm{SnO}_{2}$-based electrodes [49, 50]. For comparison, some recent representative works of $\mathrm{SnO}_{2}$-based anodes are listed (Table $\mathrm{S} 1$ ), which shows that the lithium ion storage capability of our prepared honeycomb-like composites is comparable or even better.

In order to examine the rate capability, the honeycomb-like composites electrodes were galvanostatically tested at progressively increased current densities from $50 \mathrm{~mA} \mathrm{~g}^{-1}$ to 1000 $\mathrm{mA} \mathrm{g}^{-1}$. Outstanding rate capability is demonstrated. As shown in Fig. 4e, the discharge capacities of the honeycomb-like composites electrodes are 971, 861, 750, 575 and 514 mAh $\mathrm{g}^{-1}$ at the current densities of 50 to $100,200,500$ and $1000 \mathrm{mAg}^{-1}$, respectively. Remarkably, when the current density restored to $50 \mathrm{mAg}^{-1}$ again after 50 cycles, a discharge capacity as 
high as $900 \mathrm{mAh} \mathrm{g}^{-1}$ was successfully obtained, evidencing the advance of the designed honeycomb-like structure and capability in enduring the volume expansion of $\mathrm{SnO}_{2}$ upon cycling. Additionally, the honeycomb-like composites-less also exhibits similar electrochemical rating performance, and cycling behaviors both at low current density and high current density (see Fig. S6c-e).
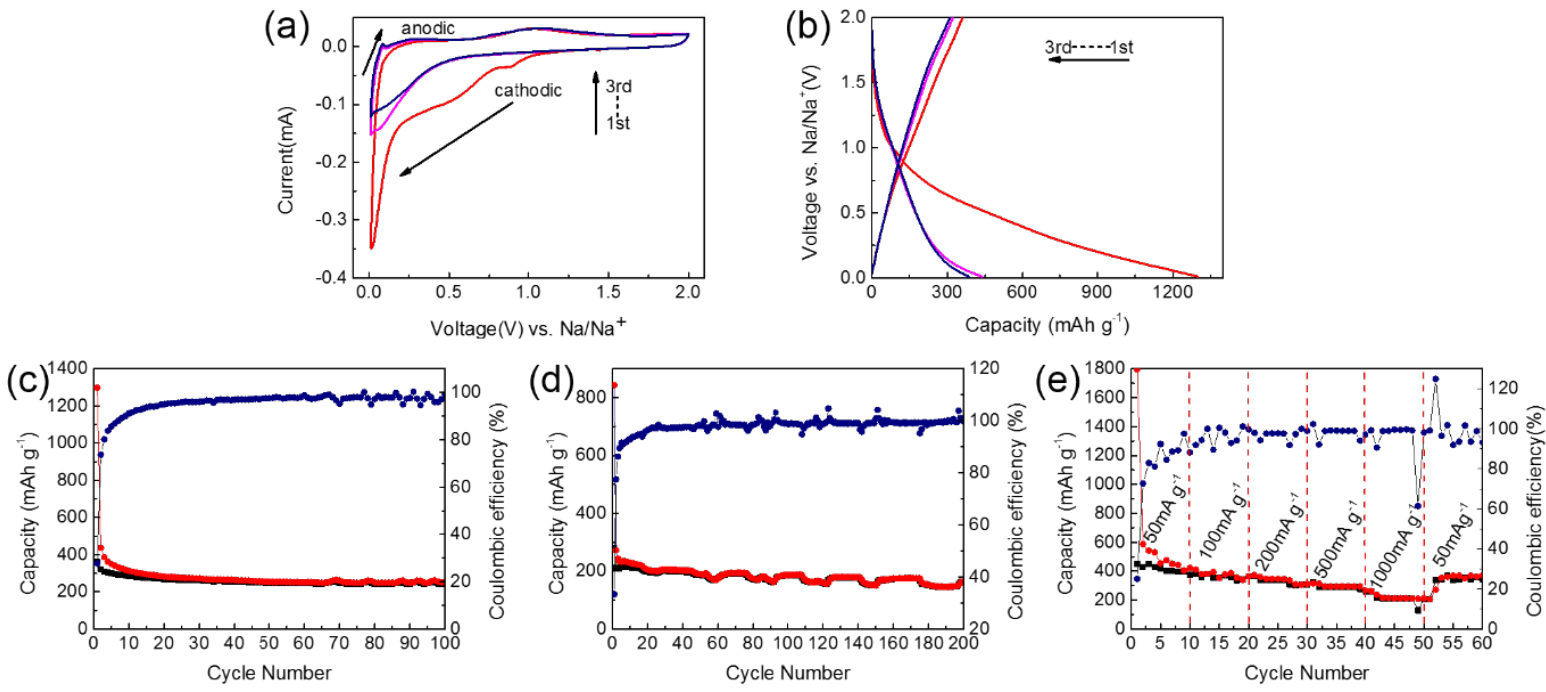

Fig. 5. (a) Cyclic voltammograms of the honeycomb-like composites anodes in SIBs; (b)

Galvanostatic charge/discharge profiles for the first three cycles of the honeycomb-like composites anodes in SIBs; (c, d) Cycling performance and Coulombic efficiency of the honeycomb-like composites anodes in SIBs at current densities of $100 \mathrm{~mA} \mathrm{~g}^{-1}$ and $500 \mathrm{~mA} \mathrm{~g}^{-1}$; (e) Rate capabilities of the honeycomb-like composites anodes in SIBs.

The sodium storage capability of the honeycomb-like composites is also explored by assembling the active materials into a coin cell using Na metal foil as the counter electrode. Fig. 5a shows the CV curves of the honeycomb-like composites electrode for the initial three cycles in the voltage range of $0-2 \mathrm{~V} v \mathrm{vs} \mathrm{Na} / \mathrm{Na}^{+}$at a scan rate of $0.1 \mathrm{mVs}^{-1}$. In the first cycle, 
the broad peak at $\sim 0.88 \mathrm{~V}$ is observed, which is associated to the reduction of $\mathrm{SnO}_{2}$ and the formation of $\mathrm{Na}_{2} \mathrm{O}$, as described by eqn.3. With discharging continues, another broad peak in the range of 0.65 to $0.37 \mathrm{~V}$ was identified, corresponding to the formation of $\mathrm{Na}_{\mathrm{x}} \mathrm{Sn}$ alloys [14, 51], which can refer to eqn. 4. After the first cycle, nearly no changes occur in all redox peaks, indicating that the irreversible reaction is mainly happened in the initial cycle [52] and the solid-electrolyte interface (SEI) films were formed on the electrode surface in the first cycle [53]. In the anodic scan, a broad oxidation peak ranging from 0.1 to $0.7 \mathrm{~V}$ is distinguished, ascribing to the dealloying reaction of $\mathrm{Na}_{\mathbf{x}} \mathrm{Sn}$, i.e. $\mathrm{NaSn}, \mathrm{NaSn}_{5}, \mathrm{Na}_{3} \mathrm{Sn}, \mathrm{Na}_{9} \mathrm{Sn}_{4}$ and $\mathrm{Na}_{15} \mathrm{Sn}_{4}$ $[54,55]$. The observed characteristics of the Na-Sn alloying/dealloying behavior are relatively analogous to the Li-Sn counterparts [56, 57].

$$
\begin{gathered}
\mathrm{SnO}_{2}+4 \mathrm{Na}^{+}+4 e^{-} \leftrightarrow \mathrm{Sn}+2 \mathrm{Na}_{2} \mathrm{O} \\
x \mathrm{Na}^{+}+\mathrm{Sn}+x e^{-} \leftrightarrow \mathrm{Na}_{x} \mathrm{Sn}
\end{gathered}
$$

The galvanostatic charge-discharge voltage profiles of the as-prepared product was collected for the initial three cycles. The test was performed at a current density of $100 \mathrm{mAg}^{-1}$ in a voltage range of $0.01-2.0 \mathrm{~V}$. Interestingly, the first discharge capacity is extremely high, being of $1298 \mathrm{mAh} \mathrm{g}^{-1}$ as shown in Fig. 5b. But the delivered charge capacity can only go back to $360 \mathrm{mAh} \mathrm{g}^{-1}$ with large irreversible capacity. This is also qualitatively analogous to the Coulombic efficiency case in Li-Sn counterparts. The reasons are speculated to be similar to the statement for the Li-Sn case that given aforementioned. Besides, another possible reason could be due to the fact that the conversion from $\mathrm{SnO}_{2}$ to $\mathrm{Sn}$ is incomplete in the initial discharging process [57]. One obvious discharge plateaus at $0.7-0.2 \mathrm{~V}$ is related to the formation of $\mathrm{Na}_{\mathrm{x}} \mathrm{Sn}$ alloys, being consistent with the CV measurement. 
To unveil the cyclic stability of the honeycomb-like composites toward sodium storage, the cycling performance was carried out at a current density of $100 \mathrm{~mA} \mathrm{~g}^{-1}$ as shown in Fig. 5c. Coinciding with that in Fig. 5b, it exhibits an ultrahigh discharging capacity with a relative low charge capacity, delivering a low Coulombic efficiency, however, it bounced back to a high value of $91 \%$ in the initial several cycles. A specific capacity of $251.5 \mathrm{mAh} \mathrm{g}^{-1}$ is delivered after 100 cycles. In comparison with the counterpart LIBs results (928.9 mAh g-1), the capacity of SIBs is $~ 3.7$ times lower, which is probably due to the larger radius size of $\mathrm{Na}$ ion $(1.02 \AA)$ than that of $\mathrm{Li}+(0.59 \AA)$ [58]. Besides, the huge volume expansion of $\sim 520 \%$ happens upon Na-Sn alloying for the formation of $\mathrm{Na}_{\mathrm{x}} \mathrm{Sn}$, together with the following aggregation of Sn fragments along cycling, leading to a rapid capacity degradation [59-61]. Because of these drawbacks, the practical application of $\mathrm{SnO}_{2}$ in $\mathrm{SIBs}$ has been severely hindered. Nonetheless, no capacity degradation has been observed after 100 cycles, verifying our designed electrode structure can well address the volume expansion upon $\mathrm{Na}^{+}$ insertion/desertion and prevent the aggregation over cycling. To highlight the robust nature of our deigned honeycomb-like structure, cycling performance at high current density of $500 \mathrm{~mA}$ $\mathrm{g}^{-1}$ was also evaluated, in which the composites experienced fiercer $\mathrm{Na}^{+}$insertion and extraction process. Again, stable cycling performance is exhibited for over 200 cycles, delivering a specific capacity of $171 \mathrm{mAh} \mathrm{g}^{-1}$, further evidencing the advance of our designed structure in the case of applications in SIBs (Fig. 5d). In order to evaluate the rate capacity of the honeycomb-like composites, a rate performance test was also implemented and shown in Fig. 5e. In the initial 10 cycles, a capacity of $469 \mathrm{mAh} \mathrm{g}^{-1}$ is achieved at a current density of $50 \mathrm{~mA} \mathrm{~g}^{-1}$. Upon increasing the current densities to $100,200,500$, and1000 $\mathrm{mA} \mathrm{g}^{-1}$, the 
discharge capacities are 370, 332, 290 and 215 mAh g $^{-1}$, respectively. After fifty cycles under different current densities, a capacity of $343 \mathrm{mAhg}^{-1}$ is recovered when the current density reduced to $50 \mathrm{mAg}^{-1}$ again. Although the delivered capacity in SIBs is significantly lower than that counterparts in LIBs, the energy storage capability is still comparable or superior to other anode candidates in SIBs and outperform the commercial utilized graphite in LIBs [62, 63]. For comparison, the cycling performance at low current density (100 mA g ${ }^{-1}$ ), high current density (500 $\mathrm{mA} \mathrm{g}^{-1}$ ), and rate capacity were all evaluated and collected as demonstrated in Fig. S7c-e, which also present high capacities and excellent stability over cycling.

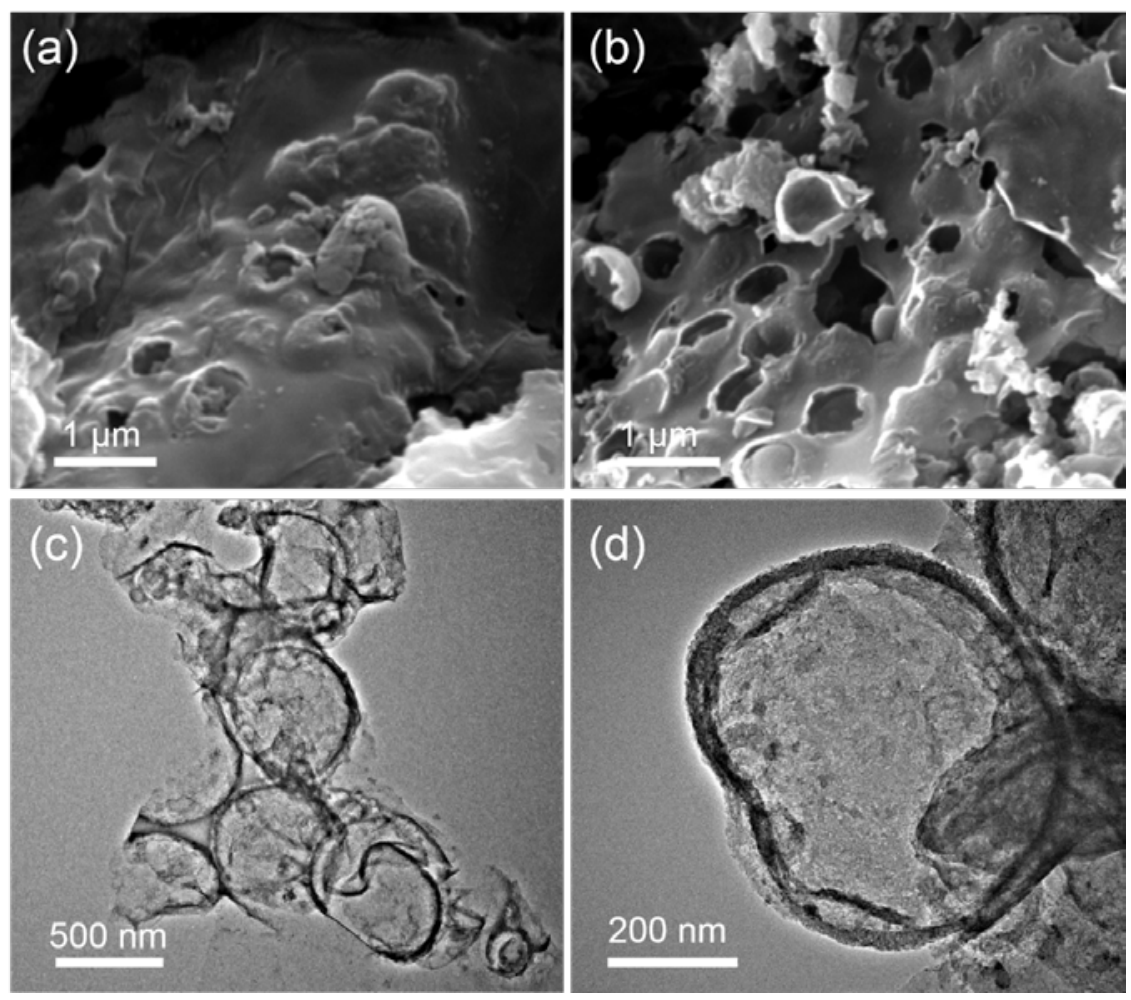

Fig. 6. (a, c) SEM and TEM images of honeycomb-like composites electrodes after 100 cycles in LIBs; (b, d) SEM and TEM images of honeycomb-like composites electrodes after 100 cycles in SIBs. 
The outstanding cyclic stability of the honeycomb-like composites in both LIBs and SIBs should be due to the following advantages of our novel designed architecture: (1) the void core/shell configuration allows accommodation of the large volume expansion during $\mathrm{Li}^{+} / \mathrm{Na}^{+}$ uptake and release process, even up to 520\%. This is supported by the SEM and TEM images that collected after 100 cycles at $100 \mathrm{~mA} \mathrm{~g}^{-1}$ shown in Fig. 6. Even after long cycling, the honeycomb feature can be still easily identified (see Fig. 6a, b), verifying the role of the designed architecture in addressing the volume expansion during $\mathrm{Li}^{+} / \mathrm{Na}^{+}$insertion and extraction processes. More close detail observation of the honeycomb-like composites was also disclosed by TEM (shown in Fig. 6c, d), where carbon encapsulated interconnected nanospheres can be still well seen; particularly, intact nanospheres are widely found in anode materials of SIB, further evidencing the robust nature of our designed architecture. (2) The carbon shell and the supported carbon films, namely carbon internetworks, prevent the happing of pulverization and aggregation during cycling. (3) The high conductive carbon networks facilitate the diffusion/transfer of both electrons and Li/Na ions (see Fig. S8); (4) The high porosity nature of the hierarchical structure enhance the contact between the electrodes and the electrolyte.

\section{Conclusion}

A novel honeycomb-like structure composing of $\mathrm{SnO}_{2} @ \mathrm{C}$ nanospheres embedded in carbon film has been fabricated using silica nanospheres and sodium chloride templates. The TGA and BET results reveal that the percentage of $\mathrm{SnO}_{2}$ in the product is $71.4 \%$, possessing $231.7 \mathrm{~m}^{2} \mathrm{~g}^{-1}$ specific surface area. Such honeycomb-like composite anodes exhibit a specific capacity of $928.9 \mathrm{mAh} \mathrm{g}^{-1}$ for LIBs and $251.5 \mathrm{mAh} \mathrm{g}^{-1}$ for SIBs, respectively, at a current 
density of $100 \mathrm{~mA} \mathrm{~g}^{-1}$ over 100 cycles without any discernable capacity degradation. The high delivered capacity, excellent stability and prominent rating capability have been achieved due to the advanced nature of the unique microstructure. The hollow $\mathrm{SnO}_{2} @ \mathrm{C}$ nanosphere offers enough space to endure the volume expansion of $\mathrm{SnO}_{2}$ during $\mathrm{Li}^{+} / \mathrm{Na}^{+}$uptake and release processes, and provides profuse pathways allowing electrolyte to contact with the electrodes. Besides, the high conductive carbon internetworks play a crucial role in improving the conductivity of the electrodes. We demonstrate that the honeycomb-like composites provide a promising protocol for engineering $\mathrm{SiO}_{2}$ as a low-cost, environmental friendly, high performance LIBs and SIBs. Moreover, our approach can also be extended for fabrication of other high volume experienced anode materials toward high-performance batteries, especially Sodium-ion batteries.

\section{Acknowledgements}

This work was financially supported by the National Natural Science Foundation of China (NSFC Grants No. 51502099, 51571096, and 61405026), Natural Science Foundation of Hubei Province (No. 2016CFB129), and "the Fundamental Research Funds for the Central Universities”, HUST: 2016YXMS211. C.D.W. acknowledges the Hubei “Chu-Tian Young Scholar” program. J.W.S. thanks the financial support from Swedish Research Councils (Vetenskapsrådet: 621-2014-5461; Formas: 2016-00559) and the Åforsk foundation (16-399). The authors appreciate the technical support from the Analytical and Testing Center of Huazhong University of Science and Technology.

\section{Appendix. Supplementary data}

Supplementary data related to this article can be found at XXX 


\section{References}

[1] B. Huang, X. Li, Y. Pei, S. Li, X. Cao, R.C. Massé, G. Cao, Small 12 (2016) 1945-1955.

[2] J.B. Goodenough, K.-S. Park, J. Am. Chem. Soc. 135 (2013) 1167-1176.

[3] C. Wang, M. Lan, Y. Zhang, H. Bian, M.-F. Yuen, K.K. Ostrikov, J. Jiang, W. Zhang, Y.Y. Li, J. Lu Green Chem., 18 (2016) 3029-3039.

[4] Y. Ruan, C. Wang, J. Jiang, J. Mater. Chem. A 4 (2016) 14509-14538.

[5] C. Wang, Y.-S. Li, J. Jiang, W.-H. Chiang, ACS Appl. Mat. Interfaces 7 (2015) 17441-17449.

[6] S. Guo, J. Yi, Y. Sun, H. Zhou, Energy Environ. Sci. 9 (2016) 2978-3006.

[7] J. Liu, P. Kopold, C. Wu, P.A. van Aken, J. Maier, Y. Yu, Energy Environ. Sci. 8 (2015) 3531-3538.

[8] P. Thomas, J. Ghanbaja, D. Billaud, Electrochim. Acta 45 (1999) 423-430.

[9] D. Stevens, J. Dahn, J. Electrochem. Soc. 148 (2001) A803-A811.

[10] C. Wang, Y. Li, Y.-S. Chui, Q.-H. Wu, X. Chen, W. Zhang, Nanoscale 5 (2013) 10599-10604.

[11] H. Wang, Q. Wu, D. Cao, X. Lu, J. Wang, M.K.H. Leung, S. Cheng, L. Lu, C. Niu, Mater. Today Energy 1-2 (2016) 24-32.

[12] K. Zhao, L. Zhang, R. Xia, Y. Dong, W. Xu, C. Niu, L. He, M. Yan, L. Qu, L. Mai, Small 12 (2016) 588-594.

[13] M. Dirican, Y. Lu, Y. Ge, O. Yildiz, X. Zhang, ACS Appl. Mat. Interfaces 7 (2015) 18387-18396.

[14] Y. Wang, D. Su, C. Wang, G. Wang, Electrochem. Commun. 29 (2013) 8-11.

[15] W. Xu, K. Zhao, C. Niu, L. Zhang, Z. Cai, C. Han, L. He, T. Shen, M. Yan, L. Qu, Nano Energy 8 (2014) 196-204.

[16] J. Wang, W. Li, F. Wang, Y. Xia, A.M. Asiri, D. Zhao, Nanoscale 6 (2014) 3217-3222.

[17] X. Zhou, L.J. Wan, Y.G. Guo, Adv. Mater. 25 (2013) 2152-2157.

[18] M. Xie, X. Sun, S.M. George, C. Zhou, J. Lian, Y. Zhou, ACS Appl. Mat. Interfaces 7 (2015) 27735-27742.

[19] W. Stöber, A. Fink, E. Bohn, J. Colloid Interface Sci. 26 (1968) 62-69.

[20] W. Li, Y. Tang, W. Kang, Z. Zhang, X. Yang, Y. Zhu, W. Zhang, C.S. Lee, Small 11 (2015) 1345-1351.

[21] M. Kruk, M. Jaroniec, Chem. Mater. 13 (2001) 3169-3183.

[22] C. Wang, M.F. Yuen, T.W. Ng, S.K. Jha, Z. Lu, S.Y. Kwok, T.L. Wong, X. Yang, C.S. Lee, S.T. Lee, Appl. Phys. Lett. 100 (2012) 253107.

[23] A. Ferrari, J. Meyer, V. Scardaci, C. Casiraghi, M. Lazzeri, F. Mauri, S. Piscanec, D. Jiang, K. Novoselov, S. Roth, Phys. Rev. Lett. 97 (2006) 187401.

[24] C. Thomsen, S. Reich, Phys. Rev. Lett. 85 (2000) 5214.

[25] A.C. Ferrari, J. Robertson, Phys. Rev. B 61 (2000) 14095.

[26] C. Wang, Y. Zhou, L. He, T.-W. Ng, G. Hong, Q.-H. Wu, F. Gao, C.-S. Lee, W. Zhang, Nanoscale 5 (2013) 600-605.

[27] A.C. Ferrari, Solid State Commun. 143 (2007) 47-57.

[28] J. Kaur, J. Shah, R. Kotnala, K.C. Verma, Ceram. Int. 38 (2012) 5563-5570.

[29] Q. Tian, Y. Tian, Z. Zhang, L. Yang, S.-i. Hirano, J. Mater. Chem. A 3 (2015) 18036-18044.

[30] C. Zhang, X. Peng, Z. Guo, C. Cai, Z. Chen, D. Wexler, S. Li, H. Liu, Carbon 50 (2012) 1897-1903.

[31] Y.D. Wang, I. Djerdj, M. Antonietti, B. Smarsly, Small 4 (2008) 1656-1660.

[32] X. Wang, J. Li, Z. Chen, L. Lei, X. Liao, X. Huang, B. Shi, J. Mater. Chem. A (2016).

[33] J.C. Lytle, H. Yan, N.S. Ergang, W.H. Smyrl, A. Stein, J. Mater. Chem. 14 (2004) 1616-1622.

[34] J. Liang, X.Y. Yu, H. Zhou, H.B. Wu, S. Ding, X.W.D. Lou, Angew. Chem. Int. Ed. 53 (2014) 12803-12807.

[35] R. Demir-Cakan, Y.-S. Hu, M. Antonietti, J. Maier, M.-M. Titirici, Chem. Mater. 20 (2008) 1227-1229. 
[36] L. Ji, Z. Lin, B. Guo, A.J. Medford, X. Zhang, Chem. - Eur. J. 16 (2010) 11543-11548.

[37] B. Guo, J. Shu, K. Tang, Y. Bai, Z. Wang, L. Chen, J. Power Sources 177 (2008) 205-210.

[38] Z.-S. Wu, Y. Sun, Y.-Z. Tan, S. Yang, X. Feng, K. Müllen, J. Am. Chem. Soc. 134 (2012) 19532-19535.

[39] L. Qie, W.M. Chen, Z.H. Wang, Q.G. Shao, X. Li, L.X. Yuan, X.L. Hu, W.X. Zhang, Y.H. Huang, Adv. Mater. 24 (2012) 2047-2050.

[40] K.H. Seng, J. Liu, Z.P. Guo, Z.X. Chen, D. Jia, H.K. Liu, Electrochem. Commun. 13 (2011) 383-386.

[41] K. Qian, B. Li, Y. Li, C. Wang, Y. Yang, Ionics 23, 2017, 1091-1096.

[42] J.H. Ku, Y.S. Jung, K.T. Lee, C.H. Kim, S.M. Oh, J. Electrochem. Soc.156 (2009) A688-A693.

[43] C. Wang, Q. Zhang, Q.-H. Wu, T.-W. Ng, T. Wong, J. Ren, Z. Shi, C.-S. Lee, S.-T. Lee, W. Zhang, RSC Adv. 2 (2012) 10680-10688.

[44] Y. Wang, F. Su, J.Y. Lee, X. Zhao, Chem. Mater. 18 (2006) 1347-1353.

[45] H. Liu, G. Wang, J. Wang, D. Wexler, Electrochem. Commun. 10 (2008) 1879-1882.

[46] J. Zhou, H. Song, X. Chen, L. Zhi, S. Yang, J. Huo, W. Yang, Chem. Mater. 21 (2009) 2935-2940.

[47] S. Jin, H. Deng, D. Long, X. Liu, L. Zhan, X. Liang, W. Qiao, L. Ling, J. Power Sources 196 (2011) 3887-3893.

[48] H. Wang, F. Fu, F. Zhang, H.-E. Wang, S.V. Kershaw, J. Xu, S.-G. Sun, A.L. Rogach, J. Mater. Chem. 22 (2012) 2140-2148.

[49] C. Wang, Y.-S. Chui, R. Ma, T. Wong, J.-G. Ren, Q.-H. Wu, X. Chen, W. Zhang, J. Mater. Chem. A 1 (2013) 10092-10098.

[50] F.F. Cao, J.W. Deng, S. Xin, H.X. Ji, O.G. Schmidt, L.J. Wan, Y.G. Guo, Adv. Mater. 23 (2011) 4415-4420.

[51] X. Xie, S. Chen, B. Sun, C. Wang, G. Wang, ChemSusChem 8 (2015) 2948-2955.

[52] Y. Zhao, J. Li, N. Wang, C. Wu, G. Dong, L. Guan, J. Phys. Chem. C 116 (2012) 18612-18617.

[53] W. Li, R. Yang, J. Zheng, X. Li, Nano Energy 2 (2013) 1314-1321.

[54] Y.-X. Wang, Y.-G. Lim, M.-S. Park, S.-L. Chou, J.H. Kim, H.-K. Liu, S.-X. Dou, Y.-J. Kim, J. Mater. Chem. A 2 (2014) 529-534.

[55] V.L. Chevrier, G. Ceder, J. Electrochem. Soc. 158 (2011) A1011-A1014.

[56] S. Li, Y. Wang, J. Qiu, M. Ling, H. Wang, W. Martens, S. Zhang, RSC Adv. 4 (2014) 50148-50152.

[57] L. Fan, X. Li, B. Yan, J. Feng, D. Xiong, D. Li, L. Gu, Y. Wen, S. Lawes, X. Sun, Adv. Energy Mater. (2016)

[58] C. Zhu, X. Mu, P.A. van Aken, Y. Yu, J. Maier, Angew. Chem. Int. Ed. 53 (2014) 2152-2156.

[59] D. Su, H.-J. Ahn, G. Wang, Chem. Commun. 49 (2013) 3131-3133.

[60] Z. Li, J. Ding, H. Wang, K. Cui, T. Stephenson, D. Karpuzov, D. Mitlin, Nano Energy 15 (2015) 369-378.

[61] S. Yang, W. Yue, J. Zhu, Y. Ren, X. Yang, Adv. Funct. Mater. 23 (2013) 3570-3576.

[62] J. Ding, H. Wang, Z. Li, A. Kohandehghan, K. Cui, Z. Xu, B. Zahiri, X. Tan, E.M. Lotfabad, B.C. Olsen, ACS nano 7 (2013) 11004-11015.

[63] Y. Cao, L. Xiao, M.L. Sushko, W. Wang, B. Schwenzer, J. Xiao, Z. Nie, L.V. Saraf, Z. Yang, J. Liu, Nano Lett. 12 (2012) 3783-3787. 